Saúde e Pesquisa, Maringá (PR)

DOI: $10.17765 / 2176-9206.2019 v 12 n 3 p 495-502$

\title{
PARASITOSES INTESTINAIS EM PACIENTES ATENDIDOS NO HOSPITAL DAS CLÍNICAS DA FACULDADE DE MEDICINA DE MARÍLIA-SP
}

\section{Paulo Sá Filizzola Martins}

Graduando do curso de medicina da Faculdade de Medicina de Marília, Brasil.

\section{Roberto Esteves Pires Castanho}

Médico. Doutor em Análises Clínicas, área de Parasitologia. Docente da disciplina de Parasitologia da Faculdade de Medicina de Marília.

\section{Eduardo Federighi Baisi Chagas}

Educador físico. Doutor em Desenvolvimento Humano e Tecnologias. Docente da disciplina de Bioestatistica da Universidade de Marília.

\section{Luciamare Perinetti Alves Martins}

Farmacêutica. Doutora em Biotecnologia aplicada a Farmácia. Docente da Disciplina de Parasitologia da Faculdade de Medicina de Marília.
RESUMO: O objetivo desta pesquisa foi verificar a prevalência de enteroparasitoses em pacientes atendidos no Hospital das Clínicas da Faculdade de Medicina de Marília-SP. Desta forma foi realizado um estudo descritivo e analítico, do tipo transversal retrospectivo, com coleta dos dados no Sistema Hospitalar Informatizado do Laboratório de Parasitologia da Faculdade de Medicina de Marília. Entre janeiro de 2012 a dezembro de 2017 foram analisadas 14.343 amostras. Os dados de prevalência foram representados por razão de prevalência e seus respectivos Intervalos de Confiança de $95 \%$. Diferenças significativas entre anos ou faixa etárias foram determinadas pela não intersecção dos limites inferiores e superiores de seus IC95\%. Observaram-se 2.149 amostras positivas para parasitas, predominando Blastocystis bominis (10,13\%), Giardia lamblia (3,35\%) e Strongyloides stercoralis $(1,16 \%)$. Pode-se inferir que a redução da prevalência das parasitoses é o resultado de políticas públicas pelo uso de medicamentos profiláticos no intuito de interromper a transmissão fecal-oral e não somente pela melhora da infraestrutura sanitária.

PALAVRAS-CHAVE: Doenças parasitárias; Giardia lamblia; Blastocystis hominis; Saneamento básico.

\section{INTESTINE PARASITOSIS IN PATIENTS AT THE HOSPITAL OF THE SCHOOL OF MEDICINE IN MARÍLIA, BRAZIL}

ABSTRACT: The prevalence of enteroparasitosis in patients at the hospital of the School of Medicine in Marilia SP Brazil, is analyzed through a descriptive, analytic, retrospective and transversal study. Data were retrieved from the Digitalized Information System of the Laboratory of Parasitology of the School of Medicine in Marilia where 14,343 patients were investigated between January 2012 and December 2017. Prevalence data were given in ranking and their respective confidence intervals at $95 \%$. Significant differences between years or age groups were determined by non-intersection of lower and higher limits of IC95\%. There were 2,149 positive samples for parasites, with a predominance of Blastocystis bominis (10.13\%), Giardia lamblia (3.35\%) and Strongyloides stercoralis (1.16\%). Results show that the decrease of parasite prevalence is due to public policies through prophylactic medicine to interrupt the fecal-oral transmission rather than by improvement of the sanitary infrastructure.

KEYWORDS: Parasitic Diseases; Giardia lamblia; Blastocystis bominis; Basic sanitation; Parasitic diseases. 


\section{INTRODUÇÃO}

A relação entre enteroparasitoses, condições sanitárias, socioeconômicas e hábitos de vida da população é bem documentada por vários autores ${ }^{1-3}$. Tendo em vista o mecanismo de transmissão dessas doenças, fatores como o acesso a saneamento básico, higiene das mãos e lavagem adequada dos alimentos são de suma importância na prevenção da ingestão de cistos e ovos dos parasitas ${ }^{4}$.

As parasitoses cursam com manifestações clínicas que incluem diarreia, má absorção, obstrução intestinal, colite, anemia e desnutrição, resultando em prejuízos ao indivíduo e à economia ${ }^{5}$. A patogenicidade varia de acordo com a espécie de parasita, a carga parasitária e condições do próprio indivíduo, como estado nutricional, doenças de base associadas e principalmente o estado imunológico ${ }^{6}$.

Embora no Brasil tenha havido melhora nas condições sanitárias nos últimos anos, poucos estudos são encontrados relatando a real prevalência de enteroparasitoses em nossa população.

No Estado de São Paulo, Anaruma-Filho et al. ${ }^{7}$ encontraram uma positividade de enteroparasitas de 59,8\% em populações domiciliares em áreas de risco de enchentes, na cidade de Campinas. Ferreira et al. ${ }^{8}$, ao analisarem a tendência secular de parasitoses na infância na cidade de São Paulo, encontraram associação entre parasitismo e nível de renda, demonstrando uma redução de $30,9 \%$ para $10,7 \%$ da prevalência de parasitoses no período de 1984 a 1996.

Com o intuito de analisar a prevalência de diferentes parasitoses em pacientes referenciados ao laboratório de Parasitologia da Faculdade de Medicina de Marília-SP (Famema), foi realizada a análise dos dados obtidos no período de janeiro de 2012 a dezembro de 2017.

\section{METODOLOGIA}

Trata-se de um estudo transversal e retrospectivo realizado no período de janeiro de 2012 a dezembro de 2017. Os dados foram obtidos no sistema SIHOSP, no ícone SDHI 1000 relatório de Laboratório, no item
SHDI2005 resultado de Exame. Somente os dados referentes ao resultado do exame parasitológico de fezes e a faixa etária foram utilizados. Foram selecionados apenas os parasitas considerados como patogênicos ao homem.

Analisaram-se 14.343 resultados de exames parasitológicos de fezes realizados no laboratório de Parasitologia da Famema, cuja área de abrangência inclui diversos municípios englobados pelo Departamento Regional de Saúde de Marília-SP (DRS IX).

Os métodos parasitológicos utilizados pelo laboratório para o diagnóstico parasitológico incluem o método direto diluído em água destilada e corado pelo lugol ${ }^{9}$, método de Faust et al. ${ }^{10}$, método de Kato modificado por Katz et al. ${ }^{11} \mathrm{e}$ Rugai et al..$^{12}$.

A análise estatística foi realizada estimando o tamanho da amostra para testar a hipótese de que houve modificação na prevalência de exames positivos para parasitoses entre os anos de 2012 a 2017. Para o cálculo do tamanho da amostra foi considerado 1 grau de liberdade, um tamanho de efeito pequeno $(0,10)$, uma margem de erro do tipo I $(\alpha)$ de $5 \%$ e um poder de estudo de $80 \%$. Deste modo, o tamanho de amostra mínimo por ano foi de 785 exames por ano, distribuídos de acordo com a demanda entre as faixas etárias de zero a seis anos, 7 a 12 anos, 13 a 21 anos, e $>22$ anos. O cálculo do tamanho da amostra foi realizado no software G*Power, version 3.1.9.2 (Franz Faul, Universität Kiel, Germany). Os dados de prevalência estão representados por razão de prevalência e seus respectivos Intervalos de Confiança de 95\% (IC95\%), representado pelo limite inferior (LiIC95\%) e limite superior (LsIC95\%). As diferenças significativas entre anos ou faixa etárias foram determinadas pela não intersecção dos limites inferiores e superiores de seus IC95\%.

O estudo foi aprovado pelo Comitê de Ética de Pesquisa da Famema, sob número 79192017.1.0000.5413.

\section{RESULTADOS}

Dentre os 14.349 exames parasitológicos de fezes analisados, observou-se que o maior número de exames ocorreu nas faixas etárias de zero a seis anos e acima de 22 anos (Tabela 1). 
Tabela 1. Distribuição da razão de prevalência (P) e intervalo de confiança de 95\% (IC) de exames positivos para parasitose por faixa etária compreendendo o período de 2012 a 2017

\begin{tabular}{|c|c|c|c|c|c|c|}
\hline \multirow[b]{2}{*}{ Ano } & & \multicolumn{4}{|c|}{ Faixa etária } & \multirow{2}{*}{$\frac{\text { Total /ano }}{\mathrm{n}}$} \\
\hline & & 0 a 6 anos & 7 a 13 anos & 13 a 21 anos & $>22$ anos & \\
\hline \multirow{2}{*}{2012} & $\mathbf{P}(\mathrm{IC})$ & $14,2(12.4 / 16,3)$ & $17,6(15,1 / 20,4)$ & $15,8(12,7 / 19,5)$ & $18,9(17,0 / 20,9)$ & \multirow{2}{*}{4006} \\
\hline & n & 1223 & 785 & 449 & 1549 & \\
\hline \multirow{2}{*}{2013} & $\mathbf{P}(\mathrm{IC})$ & $14,8(12,6 / 17,2)$ & $15,4(13,0 / 18,3)$ & $16,3(14,5 / 26,1)$ & $19,6(17,6 / 21,7)$ & \multirow{2}{*}{3404} \\
\hline & n & 935 & 712 & 325 & 1432 & \\
\hline \multirow{2}{*}{2014} & $\mathbf{P}(\mathrm{IC})$ & $11,6(10,6 / 16,1)$ & $15,0(12,3 / 18,1)$ & $13,7(10,5 / 17,7)$ & $18,4(16,5 / 20,5)$ & \multirow{2}{*}{3176} \\
\hline & n & 856 & 568 & 351 & 1401 & \\
\hline \multirow{2}{*}{2015} & $\mathbf{P}(\mathrm{IC})$ & $11,2(8,2 / 15,0)$ & $18,6(13,9 / 24,4)$ & $13,5(9,1 / 19,4)$ & $18,6(15,9 / 21,3)$ & \multirow{2}{*}{1487} \\
\hline & $\mathbf{n}$ & 331 & 210 & 171 & 775 & \\
\hline \multirow{2}{*}{2016} & $\mathbf{P}(\mathrm{IC})$ & $10,0(6,8 / 14,4)$ & $12,9(8,8 / 18,5)$ & $12,07,2$ / 19,5) & $13,4(11,0 / 16,3)$ & \multirow{2}{*}{1176} \\
\hline & n & 241 & 186 & 108 & 641 & \\
\hline \multirow{2}{*}{2017} & P (IC) & $11,9(8,1 / 17,2)$ & $13,3(9,0 / 19,2)$ & $13,3(8,2 / 20,8)$ & $14,8(12,2 / 17,8)$ & \multirow{2}{*}{1094} \\
\hline & n & 193 & 173 & 113 & 615 & \\
\hline $\begin{array}{l}\text { Total faixa } \\
\text { etária }\end{array}$ & $\mathbf{n}$ & 3779 & 2634 & 1517 & 6413 & 14343 \\
\hline
\end{tabular}

Deste total, 2.149 amostras foram positivas para parasitas, revelando uma positividade de $14,98 \%$ dos exames realizados. Dentre estes, 162 (7,54\%) indivíduos apresentaram amostras positivas para mais de uma espécie de parasita. A Tabela 2 demonstra a frequência destas parasitoses no período estudado. Nota-se que a partir de 2015 houve diminuição no número total de exames devido à finalização do convênio entre a Faculdade de Medicina de Marília e a Secretaria Municipal de Saúde.

Tabela 2. Distribuição da frequência de parasitas diagnosticados no período de 2012 a 2017

\begin{tabular}{lcccccc}
\hline Ano & 2012 & 2013 & 2014 & 2015 & 2016 & 2017 \\
\cline { 2 - 6 } Parasitas & $\mathrm{N}(\%)$ & $\mathrm{N}(\%)$ & $\mathrm{N}(\%)$ & $\mathrm{N}(\%)$ & $\mathrm{N}(\%)$ & $\mathrm{N}(\%)$ \\
\hline B. hominis & $413(10,3)$ & $372(10,9)$ & $307(9,6)$ & $164(11,0)$ & $105(8,9)$ & $93(8,5)$ \\
G. lamblia & $145(3,6)$ & $117(3,4)$ & $101(3,1)$ & $37(2,4)$ & $32(2,7)$ & $38(3,4)$ \\
E. histolytica/E. dispar & $9(0,22)$ & $6(0,17)$ & $4(0,12)$ & $6(0,40)$ & $3(0,26)$ & $1(0,09)$ \\
A. lumbricoides & $19(0,47)$ & $6(0,17)$ & $2(0,06)$ & $1(0,07)$ & 0 & 0 \\
Ancilostomídeos & $5(0,12)$ & $7(0,20)$ & 0 & $5(0,34)$ & $1(0,09)$ & $4(0,36)$ \\
H. nana & $4(0,09)$ & $4(0,11)$ & $4(0,12)$ & 0 & 0 & $1(0,09)$ \\
Taenia sp & $8(0,19)$ & $4(0,11)$ & $9(0,28)$ & $3(0,20)$ & 0 & 0 \\
E.vermiculares & $14(0,34)$ & $13(0,38)$ & $12(0,38)$ & $2(0,13)$ & $2(0,17)$ & $3(0,27)$ \\
S. stercoralis & 42 & $43(1,26)$ & $44(1,38)$ & $23(1,55)$ & $4(0,34)$ & $11(1,00)$ \\
T. trichiura & $14(0,35)$ & $7(0,20)$ & $3(0,09)$ & $1(0,07)$ & 0 & 0 \\
S. mansoni & $2(0,04)$ & $1(0,03)$ & $2(0,06)$ & 0 & $1(0,09)$ & $1(0,09)$ \\
\hline
\end{tabular}

B. hominis: Blastocystis hominis; G. lamblia: Giardia lamblia; E. histolytica/E. díspar: Entamoeba bistolytica/Entamoeba díspar;

A. lumbricóides: Ascaris lumbricoides; H. nana: Hymenolepis nana; E.vermiculares: Enterobius vermiculares; S. stercoralis: Strongyloides stercoralis; T. trichiura: Trichirus trichiura; S. mansoni: Schistosoma mansoni. 
A Tabela 3 mostra a distribuição de cada helminto por faixa etária e ano analisado. Os parasitas que não estão demonstrados não foram diagnosticados no período analisado dentro das faixas etárias e por isso foram excluídos da tabela.

Tabela 3. Distribuição da razão de prevalência (P) e intervalo de confiança de 95\% (IC) de exames positivos para cada helmintíase por faixa etária compreendendo o período de 2012 a 2017

\begin{tabular}{|c|c|c|c|c|c|}
\hline \multirow{3}{*}{ Ano } & \multirow{3}{*}{ Parasitas } & \multicolumn{4}{|c|}{ Faixa etária } \\
\hline & & 0 a 6 anos & 7 a 13 anos & 13 a 21 anos & $>22$ anos \\
\hline & & P (IC) & $\mathbf{P}(\mathrm{IC})$ & P (IC) & P (IC) \\
\hline \multirow{8}{*}{2012} & Ancilostomídeos & $0(0 / 0)$ & $0,1(0,01 / 0,7)$ & $0,4(0,1 / 1,6)$ & $0,1(0,1 / 0,5)$ \\
\hline & A. lumbricoides & $0,9(0,5 / 1,6)$ & $0,8(0,4 / 1,7)$ & $0(0 / 0)$ & $0,1(0,01 / 0,5)$ \\
\hline & E. vermicularis & $0,5(0,2 / 1,1)$ & $0,1(0,01 / 0,7)$ & $0,9(0,3 / 2,3)$ & $0,2(0,1 / 0,6)$ \\
\hline & H. nana & $0(0 / 0)$ & $0,1(0,01 / 0,7)$ & $0,2(0,01 / 1,3)$ & $0,1(0,01 / 0,5)$ \\
\hline & S. mansoni & $0(0 / 0)$ & $0(0 / 0)$ & $0(0 / 0)$ & $0,1(0,1 / 0,5)$ \\
\hline & S. stercoralis & $0,4(0,2 / 1,0)$ & $0,3(0,1 / 0,9)$ & $0,7(0,2 / 1,9)$ & $2,1(1,5 / 2,9)$ \\
\hline & Taenia sp & $0(0 / 0)$ & $0(0 / 0)$ & $0,2(0,01 / 1,3)$ & $0,5(0,2 / 0,9)$ \\
\hline & T. trichiura & $0,5(0,2 / 1,1)$ & $0,4(0,1 / 1,1)$ & $0,7(0,2 / 1,9)$ & $0,1(0,01 / 0,5)$ \\
\hline \multirow{8}{*}{2013} & Ancilostomídeos & $0(0 / 0)$ & $0(0 / 0)$ & $0(0 / 0)$ & $0,5(0,2 / 1,0)$ \\
\hline & A. lumbricoides & $0,3(0,1 / 0,9)$ & $0,3(0,1 / 1,0)$ & $0,3(0,1 / 1,7)$ & $0(0 / 0)$ \\
\hline & E. vermicularis & $0,3(0,1 / 0,9)$ & $0,8(0,4 / 1,8)$ & $0,3(0,1 / 1,7)$ & $0,2(0,1 / 0,8)$ \\
\hline & H. nana & $0,1(0,01 / 0,6)$ & $0(0 / 0)$ & $0(0 / 0)$ & $0,2(0,1 / 0,8)$ \\
\hline & S. mansoni & $0(0 / 0)$ & $0(0 / 0)$ & $0(0 / 0)$ & $0,1(0,01 / 0,4)$ \\
\hline & S. stercoralis & $0,2(0,1 / 0,8)$ & $0,3(0,1 / 1,0)$ & $0(0 / 0)$ & $2,8(2,1 / 3,8)$ \\
\hline & Taenia sp & $0(0 / 0)$ & $0(0 / 0)$ & $0(0 / 0)$ & $0,3(0,1 / 0,7)$ \\
\hline & T. trichiura & $0,2(0,1 / 0,8)$ & $0,7(0,3 / 1,6)$ & $0(0 / 0)$ & $0(0 / 0)$ \\
\hline \multirow{7}{*}{2014} & A. lumbricoides & $0,2(0,1 / 0,8)$ & $0(0 / 0)$ & $0(0 / 0)$ & $0(0 / 0)$ \\
\hline & E. vermicularis & $0,8(0,4 / 1,7)$ & $0,5(0,2 / 1,5)$ & $0(0 / 0)$ & $0,1(0,01 / 0,5)$ \\
\hline & H. nana & $0(0 / 0)$ & $0(0 / 0)$ & $0(0 / 0)$ & $0,3(0,1 / 0,7)$ \\
\hline & S. mansoni & $0(0 / 0)$ & $0(0 / 0)$ & $0(0 / 0)$ & $0,1(0,01 / 0,5)$ \\
\hline & S. stercoralis & $0,4(0,1 / 1,0)$ & $0,2(0,01 / 1,0)$ & $0,3(0,1 / 1,6)$ & $2,9(2,1 / 3,9)$ \\
\hline & Taenia sp & $0(0 / 0)$ & $0(0 / 0)$ & $0(0 / 0)$ & $0,6(0,3 / 1,2)$ \\
\hline & T. trichiura & $0,1(0,01 / 0,7)$ & $0,2(0,01 / 1,0)$ & $0,3(0,1 / 1,6)$ & $0(0 / 0)$ \\
\hline \multirow{6}{*}{2015} & Ancilostomídeos & $0(0 / 0)$ & $0,5(0,1 / 2,6)$ & $0(0 / 0)$ & $0,5(0,2 / 1,3)$ \\
\hline & A. lumbricoides & $0(0 / 0)$ & $0,5(0,1 / 2,6)$ & $0(0 / 0)$ & $0(0 / 0)$ \\
\hline & E. vermicularis & $0,3(0,1 / 1,7)$ & $0(0 / 0)$ & $0,6(0,1 / 3,3)$ & $0(0 / 0)$ \\
\hline & S. stercoralis & $0,6(0,2 / 2,2)$ & $0,5(0,1 / 2,6)$ & $0,6(0,1 / 3,3)$ & $2,5(1,6 / 3,8)$ \\
\hline & Taenia sp & $0(0 / 0)$ & $0(0 / 0)$ & $0(0 / 0)$ & $0,4(0,1 / 1,1)$ \\
\hline & T. trichiura & $0(0 / 0)$ & $0(0 / 0)$ & $0(0 / 0)$ & $0,1(0,01 / 0,7)$ \\
\hline \multirow{4}{*}{2016} & Ancilostomídeos & $0(0 / 0)$ & $0(0 / 0)$ & $0(0 / 0)$ & $0,2(0,01 / 0,9)$ \\
\hline & E. vermicularis & $0(0 / 0)$ & $0(0 / 0)$ & $0,9(0,2 / 5,1)$ & $0,2(0,01 / 0,9)$ \\
\hline & S. mansoni & $0(0 / 0)$ & $0(0 / 0)$ & $0(0 / 0)$ & $0,2(0,01 / 0,9)$ \\
\hline & S. stercoralis & $0,4(0,1 / 2,3)$ & $0(0 / 0)$ & $0(0 / 0)$ & $0,5(0,2 / 1,4)$ \\
\hline \multirow{5}{*}{2017} & Ancilostomídeos & $0(0 / 0)$ & $0(0 / 0)$ & $2,7(0,9 / 7,5)$ & $0,2(0,01 / 0,9)$ \\
\hline & E. vermicularis & $1,0(0,3 / 3,7)$ & $0(0 / 0)$ & $0(0 / 0)$ & $0,2(0,01 / 0,9)$ \\
\hline & H. nana & $0(0 / 0)$ & $0(0 / 0)$ & $0(0 / 0)$ & $0,2(0,01 / 0,9)$ \\
\hline & S. mansoni & $0(0 / 0)$ & $0(0 / 0)$ & $0(0 / 0)$ & $0,2(0,01 / 0,9)$ \\
\hline & S. stercoralis & $0(0 / 0)$ & $0(0 / 0)$ & $0(0 / 0)$ & $1,8(1,0 / 3,2)$ \\
\hline
\end{tabular}

A. lumbricoides: Ascaris lumbricoides; H. nana: Hymenolepis nana; E.vermiculares: Enterobius vermiculares; S. stercoralis: Strongyloides stercoralis; T. trichiura: Trichirus trichiura; S. mansoni: Schistosoma mansoni.

A distribuição dos protozoários dentro de cada faixa etária e ano estudado está demonstrado na Tabela 4. 
Tabela 4. Distribuição da razão de prevalência (P) e intervalo de confiança de $95 \%$ (IC) de exames positivos para cada protozoose por faixa etária compreendendo o período de 2012 a 2017

\begin{tabular}{|c|c|c|c|c|c|}
\hline & \multirow{2}{*}{ Ano Parasitas } & \multicolumn{4}{|c|}{ Faixa etária } \\
\hline & & 0 a 6 anos & 7 a 13 anos & 13 a 21 anos & $>22$ anos \\
\hline & & P (IC) & P (IC) & P (IC) & P (IC) \\
\hline \multirow{3}{*}{2012} & B. bominis & $4,5(3,5 / 5,8)$ & $11,7(9,7 / 14,2)$ & $11,4(8,7 / 14,6)$ & $13,9(12,2 / 15,7)$ \\
\hline & E. bistolytica/E. dispar & $0,2(0,1 / 0,7)$ & $0,4(0,1 / 0,7)$ & $0(0 / 0)$ & $0,2(0,1 / 0,6)$ \\
\hline & G. lamblia & $7,2(5,9 / 8,8)$ & $3,7(2,6 / 5,3)$ & $1,3(0,6 / 2,9)$ & $1,4(0,9 / 2,1)$ \\
\hline \multirow{3}{*}{2013} & B. hominis & $5,5(4,2 / 7,1)$ & $10,5(8,5 / 13,0)$ & $13,8(10,5 / 18,0)$ & $14,0(12,3 / 15,9)$ \\
\hline & E. bistolytica/E. dispar & $0(0 / 0)$ & $0(0 / 0)$ & $0,3(0,1 / 1,7)$ & $0,3(0,1 / 0,8)$ \\
\hline & G. lamblia & $8,1(6,5 / 10,1)$ & $2,8(1,8 / 4,3)$ & $1,5(0,7 / 3,6)$ & $1,1(0,7 / 1,8)$ \\
\hline \multirow{3}{*}{2014} & B. bominis & $3,5(2,5 / 5,0)$ & $10,0(7,8 / 12,8)$ & $11,7(8,7 / 15,5)$ & $12,8(11,1 / 14,6)$ \\
\hline & E. bistolytica/E. dispar & $0(0 / 0)$ & $0(0 / 0)$ & $0,6(0,2 / 2,1)$ & $0,1(0,01 / 0,5)$ \\
\hline & G. lamblia & $6,5(5,1 / 8,4)$ & $4,0(2,7 / 6,0)$ & $0,9(0,3 / 2,5)$ & $1,4(0,9 / 2,2)$ \\
\hline \multirow{3}{*}{2015} & B. bominis & $3,9(2,3$ / 6,6) & $12,9(9,0 / 18,1)$ & $10,5(6,8 / 16,0)$ & $13,7(11,4 / 16,3)$ \\
\hline & E. bistolytica/E. dispar & $0(0 / 0)$ & $0,5(0,1 / 2,6)$ & $1,2(0,3 / 4,2)$ & $0,4(0,1 / 1,1)$ \\
\hline & G. lamblia & $6,3(4,2 / 9,5)$ & $3,8(1,9 / 3,7)$ & $0,6(0,1 / 3,3)$ & $0,9(0,4 / 1,9)$ \\
\hline \multirow{3}{*}{2016} & B. bominis & $3,3(1,7 / 6,4)$ & $9,7(6,2 / 14,8)$ & $11,1(6,5 / 18,4)$ & $10,5(8,3 / 13,1)$ \\
\hline & E. bistolytica/E. dispar & $0(0 / 0)$ & $0(0 / 0)$ & $0(0 / 0)$ & $0,5(0,2 / 1,4)$ \\
\hline & G. lamblia & $6,2(3,8 / 10,0)$ & $3,8(1,8 / 7,6)$ & $0(0 / 0)$ & $1,6(0,8 / 2,8)$ \\
\hline \multirow{3}{*}{2017} & B. bominis & $3,1(1,4 / 6,6)$ & $4,0(2,0 / 8,1)$ & $7,1(3,6 / 13,4)$ & $11,7(9,4 / 14,5)$ \\
\hline & E. bistolytica/E. dispar & $0(0 / 0)$ & $0(0 / 0)$ & $0,9(0,2 / 4,8)$ & $0(0 / 0)$ \\
\hline & G. lamblia & $7,8(4,8 / 12,4)$ & $9,2(5,8 / 14,5)$ & $2,7(0,9 / 7,5)$ & $0,7(0,3 / 1,7)$ \\
\hline
\end{tabular}

B. hominis: Blastocystis hominis; G. lamblia: Giardia lamblia; E. histolytica/E. díspar: Entamoeba histolytica/Entamoeba díspar.

\section{DISCUSSÃO}

As parasitoses intestinais afetam pessoas de todas as idades e sexos, podendo comprometer a capacidade produtiva de milhares de indivíduos ${ }^{13}$. No entanto, a morbimortalidade é maior entre a população pediátrica, sobretudo quando acomete menores de um ano de idade em países subdesenvolvidos. Assim, quadros de diarreia desencadeados por infecções parasitárias são causas frequentes de internações hospitalares ${ }^{14}$.

Os resultados obtidos nesse estudo evidenciaram o predomínio de Blastocystis hominis, Giardia lamblia e Strongyloides stercoralis sobre os demais parasitas, representando, respectivamente, 10,13\%, 3,35\% e 1,16\% do total das amostras analisadas, enquanto os outros parasitas diagnosticados somaram apenas $1,35 \%$ do total de exames realizados.
A elevada positividade para Blastocystis hominis encontrada neste estudo corrobora resultados anteriores obtidos por Martins et al. ${ }^{15}$. Neste contexto, pesquisas de Subirats e Borrás ${ }^{16}$ e Sekar e Shanthi ${ }^{17}$ relataram que o $B$. bominis é o protozoário mais comum, principalmente nos países em desenvolvimento. De acordo com Kurt et al. ${ }^{18}$ não há dúvidas quanto à patogenicidade do $B$. hominis, a qual se caracteriza por um quadro de gastroenterite, dor abdominal, prurido anal, flatulência, meteorismo, náusea, vômito e diarreia, sem presença de leucócitos ou sangue nas fezes ${ }^{19-21}$, embora estas sintomatologias fiquem na dependência de alguns fatores como interações com o hospedeiro e microbiota, além da imunidade do hospedeiro ${ }^{18}$. Apesar do potencial de causar morbidade significativa em populações expostas a este agente, em alguns casos pode haver cura espontânea ${ }^{22}$.

Os resultados mostraram alta prevalência de G. lamblia, principalmente em menores de seis anos, reforçando a necessidade de medidas higiênicas esanitárias 
que possam reduzir essa protozoonose, sobretudo em creches e escolas, locais de alta transmissibilidade pela aglomeração de crianças, uma vez que a infecção pode ocorrer de pessoa-a-pessoa, como também por água e alimentos contaminados ${ }^{23}$.

Além dessas formas de transmissão, alguns autores consideram a giardíase como uma zoonose, considerandoaimportânciadoscãescomodisseminadores da doença aos homens ${ }^{24,}{ }^{25}$. Estudos de Traub et al. ${ }^{24}$ demonstraram por testes moleculares a semelhança das espécies de Giardia de humanos e animais, e pesquisa de Volotão et al. ${ }^{26}$ reforçaram o potencial zoonótico da giardíase, quando observaram infecções com genótipos semelhantes em humanos e cães em um município do noroeste paulista.

A diminuição na prevalência das helmintíases vem sendo demonstrada por vários autores e os resultados obtidos neste estudo corroboraram várias pesquisas ${ }^{8,28}$.

Reafirmando estas pesquisas, resultados de um levantamento parasitológico realizado em escolares deste município há 30 anos atrás ${ }^{28}$, encontraram taxas bastante altas de parasitismo como 15,2\% Trichuris trichiura, $13,3 \%$ Ascaris lumbricoides e $14,4 \%$ G. lamblia com total de 40,8\% crianças parasitadas. Porém, em 2007 neste mesmo município, Martins et al. ${ }^{15}$ mostraram uma taxa de $16,7 \%$ para B. bominis e 5,6 \% para G. lamblia, sendo que as demais parasitoses apresentaram prevalência abaixo de 1,0\% exceto para Trichuris trichiura e $S$. stercoralis que apontaram 1,3\% e 1,6\% respectivamente, considerando-se todas as faixas etárias.

Assim, observou-se nesta pesquisa a redução na prevalência das parasitoses, que tem sido mais acentuada nos últimos três a quatro anos. B. hominis e G. lamblia continuam sendo os parasitas mais frequentes e $S$. stercoralis o mais prevalente entre os helmintos. Observou-se que desde 2015 não houve diagnóstico de Ascaris lumbricoides nas faixas etárias de zero a seis e sete a 13 anos, e que nas faixas etárias acima de 13 anos este parasita deixou de ser detectado desde 2013. Resultados semelhantes foram obtidos em relação ao Trichuris trichiura.

Considerando que esses parasitas são geohelmintos obrigatórios, pode-se inferir que a instalação de redes de esgotos e água tratada, mesmos nos bairros de periferia do município dificultou o contato dos indivíduos com os parasitas. De acordo com Ludwig et al. ${ }^{29}$ existe uma relação direta entre saneamento básico e a prevalência de parasitoses intestinais.

Neste contexto, cabe discutir as medidas adotadas pelas unidades de saúde com a administração de antiparasitários, seguindo a recomendação do Ministério da Saúde ${ }^{30}$, na qual a partir de 2013, as crianças em idade escolar deveriam receber tratamento com $400 \mathrm{mg}$ de albendazol em dose única como medida profilática, com o objetivo de reduzir a infecção por geo-helmintos.

Entretanto, pode se demonstrar a importância do meio peridomiciliar como disseminador do parasita ${ }^{3}$, considerando a prevalência do $S$. stercoralis, que em 1988 estava com 2,4\%, em 2007 com 1,6\%, sendo hoje com $1,16 \%$.

Talvez o uso desse medicamento preventivo não seja o mais adequado para este parasita uma vez que seriam necessárias três doses consecutivas de albendazol para alcançar a eficácia terapêutica, além da necessidade de implementação de saneamento básico e aquisição de educação sanitária pela população ${ }^{28}$.

\section{CONCLUSÃO}

Por último, ponderamos que o presente estudo não pode ser usado como representativo da prevalência de enteroparasitoses na população geral, uma vez que as amostras utilizadas são restritas a pacientes ambulatoriais ou internados que, por motivos diversos, foram referenciados ao serviço ambulatorial ou hospitalar da Famema. Ainda sim, os valores encontrados são próximos a alguns levantamentos epidemiológicos, podendo ser utilizado como uma estimativa da prevalência das parasitoses estudadas no município de Marília e região.

\section{REFERÊNCIAS}

1. World Health Organization. Soil-transmitted helminth infections: fact sheet Geneva: WHO; 2016.

2. Camello JT, Cavagnolli NI, Spada PKWDS, Poeta J, Rodrigues $\mathrm{AD}$. Prevalência de parasitoses intestinais e condições de saneamento básico das moradias em escolares da zona urbana de Caxias do Sul, Rio 
Grande do Sul. Sci med (Porto Alegre, Online). 2016;26(1):21716-.

3. Pioli M, Pires RH, Ramos SB, Martins CH, Oliveira LE, Zaia JE. Influência de fatores de risco na mortalidade por doenças infecciosas e parasitárias. Saúde e Pesquisa. 2016;9(3):491-8.

4. Motta MEFA, Silva GAP. Diarréia por parasitas. Rev Bras Saúde Mater Infant. 2002;2:117-27.

5. Colli CM, Mizutani AS, Martins VA, Ferreira EC, Gomes ML. Prevalence and risk factors for intestinal parasites in food handlers, southern Brazil. Int J Environ Health Res. 2014;24:450-8.

6. Neves D. Parasitologia humana. 12 ed. São Paulo: Atheneu; 2012.

7. Anaruma Filho F, Corrêa CRS, Ribeiro MCSA, Chieffi PP. Parasitoses intestinais em áreas sob risco de enchente no município de Campinas, estado de São Paulo, Brasil. Rev Patol Trop. 2007;36:12.

8. Ferreira MU, Ferreira CS, Monteiro CA. Tendência secular das parasitoses intestinais na infância na cidade de São Paulo (1984-1996). Rev Saúde Pública. 2000;34:73-82.

9. Amato Neto V, Correa L. Exame parasitologico de fezes. São Paulo: Sarvier; 1991.

10. Faust EC, Sawitz W, Tobie J, Odom V, Peres C, Lincicome DR. Comparative Efficiency of Various Technics for the Diagnosis of Protozoa and Helminths in Feces. J Parasitol. 1939;25:241-62 pp.

11. Katz N, Chaves A, Pellegrino J. A simple device for quantitative stool thick-smear technique in Schistosomiasis mansoni. Rev Inst Med Trop Sao Paulo. 1972;14:397-400.

12. Rugai E, Mattos T, Brisola AP. Nova técnica para isolar larvas de mematóides das fezes-Modificação do Método de Baermann. Rev Inst Adolfo Lutz. 1954;14:5-8.

13. Sousa ACM, Bocardi MIB. Hábitos de vida como fator desencadeante a parasitoses intestinais. Ideias e Inovação-Lato Sensu. 2015;2:77-92.
14. Agudelo-Lopez S, Gómez-Rodríguez L, Coronado X, Orozco A, Valencia-Gutierrez CA, Restrepo-Betancur LF, et al. Prevalencia de Parasitosis Intestinales y Factores Asociados en un Corregimiento de la Costa Atlántica Colombiana. Rev Salud Pública. 2008;10:633-42.

15. Martins LPA, Serapião AATB, Valenciano RF, Pires JECPEC, Castanho REP. Frequência de Blastocystis hominis e outras enteroparasitoses em amostras fecais analisadas no Laboratório de Parasitologia da Faculdade de Medicina de Marília-SP. Rev Patol Trop. 2007;36(1):47-53.

16. Subirats M, Borrás R. Blastocystis sp., an emerging parasite with controversial pathogenicity.

Should all human cases be treated? Rev Clín Esp. 2018;218:133-4.

17. Sekar U, Mariappan S. Blastocystis: Consensus of treatment and controversies. Trop Parasitol. 2013;3:35-9.

18. Kurt O, Doğruman Al F, Tanyüksel M. Eradication of Blastocystis in humans: Really necessary for all? Parasitol Int. 2016;65:797-801.

19. Cheng HS, Haung ZF, Lan WH, Kuo TC, Shin JW. Epidemiology of Blastocystis hominis and other intestinal parasites in a Vietnamese female immigrant population in southern Taiwan. Kaohsiung J Med Sci. 2006;22:166-70.

20. Ertug S, Karakas S, Okyay P, Ergin F, Oncu S. The effect of Blastocystis hominis on the growth status of children. Med Sci Monit. 2007;13(1):CR4D-3.

21. Velásquez V, Caldera R, Wong W, Cermeño G, Fuentes M, Blanco Y, et al. Elevada prevalência de blastocistose em pacientes do Centro de Saúde de Soledad, Estado Anzoátegui, Venezuela. Rev Soc Bras Med Trop. 2005;38:356-7.

22. Hellemond JJ, Molhoek N, Koelewijn R, Wismans P, Genderen PJJ. Is paromomycin the drug of choice for eradication of Blastocystis in adults? J Infect Chemother. 2012;19:545-8.

23. Gurgel RQ, Cardoso GdS, Silva ÂM, Santos LNd, Oliveira RCVd. Creche: ambiente expositor ou 
protetor nas infestações por parasitas intestinais em Aracaju, SE. Rev Soc Bras Med Trop. 2005;38:267-9.

24. Traub R, Monis P, Robertson I, Irwin P, Mencke N, C A Thompson R. Epidemiological and molecular evidence supports the zoonotic transmission of Giardia among humans and dogs living in the same community. Parasitology. 2004;128:253-62.

25. Capelli G, Paoletti B, Iorio R, Regalbono A, Pietrobelli M, Bianciardi P, et al. Prevalence of Giardia spp. in dogs and humans in Northern and Central Italy. Parasitol Res. 2003;90 Suppl 3:S154-5.

26. Volotão ACC, Ramos NMD, Fantinatti M, Moraes MVPd, Atique Netto H, Storti-Melo LM, et al. Giardiasis as zoonosis: between proof of principle and paradigm in the Northwestern region of São Paulo State, Brazil. Braz J Infect Dis. 2011;15:382-3.

27. Frei F, Juncansen C, Ribeiro-Paes JT. Levantamento epidemiológico das parasitoses intestinais: viés analítico decorrente do tratamento profilático. Cad Saúde Pública. 2008;24:2919-25.

28. Castanho REP, Martins LPA, Freire Junior GA, Alves AMC. Prevalencia de parasitoses intestinais em escolares do município de Marília-SP. VIII Jornada Paulista de Parasitologia; 25 a26 de maio de 1990; Campinas-SP: UNICAMP; 1990.

29. Ludwig KM, Frei F, Alvares Filho F, Ribeiro-Paes JT. Correlação entre condições de saneamento básico e parasitoses intestinais na população de Assis, Estado de São Paulo. Rev Soc Bras Med Trop. 1999;32:54755.

30. Ministerio da Saude (BR). Informe Técnico, Campanha Nacional de Hanseníase, GeoHelmintíases e Tracoma 2014. Brasilia (DF): Ministerio da Saúde; 2014.

Recebido em: 31/01/2019

Aceito em: 22/08/2019 